\title{
Comparison of Exponential Smoothing Models for Forecasting Cassava Production
}

\author{
O.V. Oni ${ }^{*}$ and Y.O. Akanle ${ }^{2}$ \\ ${ }^{1}$ Department of Statistics, Federal College of Animal Health and Production Technology, Ibadan \\ ${ }^{2}$ Department of Statistics, Federal College of Animal Health and Production Technology, Ibadan
}

Available online at: www.isroset.org

Received: 31/May/2018, Revised: 07/Jun/2018/2018, Accepted: 18/Jun/2018, Online: 30/Jun/2018

\begin{abstract}
This paper evaluated and compared the performance of a family of smoothing models such as Simple Exponential Smoothing (SES), Holt's Linear Trend (HLT), Exponential trend (ET) and Holt's Damped Methods: additive and multiplicative; to forecast the annual Cassava production in Nigeria. The predictive capabilities were compared in terms of Root Mean Square Error (RMSE), Mean Absolute Error (MAE), Mean Percentage Error (MPE) and Mean Absolute Percentage Error (MAPE) based on the validated data set. The Holt's Exponential Trend with parameter $\alpha=0.8556$ and $\beta=0.0001$ was found to have best described the data having the lowest ranked error statistics in an out of sample performance.
\end{abstract}

Keywords- Cassava, Simple Exponential Smoothing, Holt's Linear Trend, Exponential Trend, Holt's Damped Additive and Holt's Damped Multiplicative

\section{INTRODUCTION}

Cassava production is vital to Nigeria's economy as a country, it is a major producer of the commodity; it is produced in 24 of the country's 36 states. Cassava is one of the most important crops cultivated by each household in Nigeria. It has huge cost and return comparative production advantage over other staple crops.

Cassava production requires less labour per unit of output and low fertile soils for better yield. It is a good staple food, with nationally required food security minimum of 2400 calories per person per day [1].

Its root starch are used in a wide array of industries, including food manufacturing, pharmaceuticals, textiles, plywood, paper, adhesives, and as feedstock for the production of ethanol biofuel. [2]

There are relatively few studies done on forecast of annual Cassava production in developing nations such as Nigeria, [3] employed Box-Jenkin model and Exponential Smoothing Method to forecast short-term market price of cassava crop in Oyo State, Nigeria. The analysis revealed that Simple Exponential Smoothing is the best method to predict price of Cassava out of Holts' Trend, Winter's Multiplicative and Winter's Additive models used.

In comparison of several exponential smoothing techniques to forecast, the Indonesia palm oil production [4] showed that triple exponential smoothing additives model had lowest error rate compared to the other models with root mean square error.

In attempting to forecast univariate time series data, it is generally accepted that parsimonious model techniques are followed. Exponential smoothing techniques are simple tools for smoothing and forecasting a time series data. Smoothing a time series aims at eliminating the irrelevant noise and extracting the general path followed by the series [5]. This study aimed to compare the predictability performance of different exponential smoothing models to forecast Cassava production in Nigeria.

\section{METHOD OF ANALYSIS}

The data used in this study is the yearly Cassava production in Nigeria obtained from Food and Agriculture Organization Statistical Database and it covers a period of fifty-four years (1961 - 2014).

Several methods of modelling time series data were developed, notable among these models are the simple moving average, exponential smoothing, trend analysis and the ARIMA models, for the purpose of this study the exponential smoothing methodology will be applied.

The exponential smoothing models do not explicitly involve the processes of model identification and parameter estimation unlike ARIMA. Exponential smoothing methods seek to isolate trends or seasonality from irregular variation, where such patterns are present methods, that are more advanced are used. [6] 
Exponential smoothing describes a class of forecasting methods for continually revising a forecast in the light of more recent experience. The word "exponential smoothing" reveals that the weights decrease exponentially as the observation becomes older. [7]

\section{II.1 Simple Exponential Smoothing (SES)}

The relationship that characterized the simple exponential smoothing procedure is

$$
X_{t}=a+e_{t}
$$$$
1
$$

where a is constant and $e_{t}$ the residual. To forecast the next period $t+1$ in period the following series is computed recursively

$$
\hat{X}_{t+1}=\alpha X_{t}+(1-\alpha) \hat{X}_{t}
$$

Simple exponential smoothing requires only the most recently observed value of the time series and value for $\alpha$ which lies between 0 and 1. The value of $\alpha$ is usually determined by minimizing the sum of squares of the forecast errors:

$$
\frac{1}{T} \sum_{t=0}^{T-1}\left(X_{t+1}-\hat{X}_{t+1}\right)^{2}=\frac{1}{T} \sum_{t=0}^{T-1} e_{t+1}^{2} \quad 3
$$

Equation 2 when applied recursively for each observation from the series, each new smoothed value $\hat{X}_{t+1}$ is computed as the weighted average of the current observation, $X_{t}$ and the previous smoothed observation, $\hat{X}_{t}$. Thus each smoothed value $\hat{X}_{t+1}$ is the weighted average of the previous $\mathrm{n}$ observations, the weights of these decrease exponentially in the past, so $X_{1}$ has a weight of $\alpha(1-$ $\alpha)^{n-1}, X_{2}$ a weight of $\alpha(1-\alpha)^{n-2}, X_{t-1}$ being weighted with $\alpha(1-\alpha)$. So equation (2) can be written as:

$$
\hat{X}_{t+1}=\alpha \sum_{t=1}^{T}(1-\alpha)^{t} \hat{X}_{t+1-s}
$$

The initial value of $\hat{X}_{1}$ is usually equal to $X_{1}$, or with the average of the initial values of the series.

\section{II.2 Holt's linear trend method (HLT)}

The simple exponential smoothing extended by Holt allows for forecasting of data with a trend. This method involves a forecast equation and two smoothing equations [8]:

$$
\begin{array}{ll}
\hat{X}_{t}(m)=S_{t}+m T_{t} & 5 \\
S_{t}=\alpha X_{t}+(1-\alpha)\left(S_{t-1}+T_{t-1}\right) & 6 \\
T_{t}=\gamma\left(S_{t}+S_{t-1}\right)+(1-\gamma) T_{t-1} & 7
\end{array}
$$

where $X_{t}$ is the actual observation, $\hat{X}_{t}(m)$ is the $m$-stepahead forecast, $S_{t}$ an estimate of the level of the series at time $t, T_{t}$ an estimate of the trend (slope) of the series at time $t, \alpha$ is the smoothing parameter for the level, $0 \leq \alpha \leq$ 1 and $\gamma$ is the smoothing parameter for the trend.

The error correction form of the level and the trend equations show the adjustments in terms of the withinsample one-step forecast error:

$$
\begin{array}{ll}
S_{t}=S_{t-1}+T_{t-1}+\alpha e_{t} & 8 \\
T_{t}=T_{t-1}+\alpha \gamma e_{t} & 9
\end{array}
$$

where $e_{t}=X_{t}-\left(S_{t-1}+T_{t-1}\right)=X_{t}-\hat{X}_{t}$

\section{II.3 Exponential Trend method (ET)}

It is a modification of Holt's trend method, by allowing the level and the slope to be multiplied rather than added:

$$
\begin{array}{ll}
\hat{X}_{t}(m)=S_{t} R_{t}^{m} & 10 \\
S_{t}=\alpha X_{t}+(1-\alpha)\left(S_{t-1} R_{t-1}\right) & 11 \\
R_{t}=\gamma \frac{s_{t}}{S_{t-1}}+(1-\gamma) R_{t-1} & 12
\end{array}
$$

where $R_{t}$ now represents an estimated growth rate which is multiplied rather added to the estimated level. The trend in the forecast function is now exponential rather than linear, so that the forecast project a constant growth rate rather than a constant slope. The error correction form is

$$
\begin{gathered}
S_{t}=S_{t-1} R_{t-1}+\alpha e_{t} \\
R_{t}=R_{t-1}+\alpha \gamma \frac{e_{t}}{S_{t-1}}
\end{gathered}
$$

where $e_{t}=X_{t}-\left(S_{t-1} R_{t-1}\right)=X_{t}-\hat{X}_{t}$

\section{II.4 Holt's Additive Damped Trend}

Gardner and McKenzie describe how a damping parameter $0<\phi<1$, can be used within Holt's method to give more control over trend extrapolation; in conjunction with the smoothing parameters $\alpha$ and $\beta$ with values between 0 and 1 :

$$
\begin{array}{ll}
\hat{X}_{t}(m)=S_{t}+\sum_{i=1}^{m} \phi^{i} T_{t} & 15 \\
S_{t}=\alpha X_{t}+(1-\alpha)\left(S_{t-1}+\phi T_{t-1}\right) & 16 \\
T_{t}=\gamma\left(S_{t}-S_{t-1}\right)+(1-\gamma) \phi T_{t-1} & 17
\end{array}
$$

If $\phi=1$ the method is identical to Holt's linear method. For values between 0 and $1, \phi$ dampens the trend so that it approaches a constant sometime in the future. In fact, the forecasts converge to $S_{T}+\phi T_{T} /(1-\phi)$ as $\mathrm{m} \rightarrow \infty$ for any value $0<\phi<1$. The effect of this is that short-run forecasts are trended while long-run forecasts are constant. 
The error correction form of the smoothing equations is

$$
\begin{array}{ll}
S_{t}=S_{t-1}+\phi T_{t-1}+\alpha e_{t} & 18 \\
T_{t}=\phi T_{t-1}+\alpha \gamma e_{t} & 19
\end{array}
$$

\section{II.5 Holt's Multiplicative Damped Trend}

Motivated by the improvements in forecasting performance seen in the additive damped trend case, Taylor introduced a damping parameter to the exponential trend method resulting to a multiplicative damped trend method [10]:

$$
\begin{array}{ll}
S_{t}=\alpha X_{t}+(1-\alpha)\left(S_{t-1} R_{t-1}^{\phi}\right) & 20 \\
R_{t}=\gamma\left(\frac{S_{t}}{S_{t-1}}\right)+(1-\gamma) R_{t-1}^{\phi} & 21 \\
\hat{X}_{t}(m)=S_{t} R_{t}^{\sum_{i=1}^{m} \phi} & 22
\end{array}
$$

The forecast function in expression (22) is analogous to the forecast function for damped Holt's Exponential Smoothing. The growth rate undergoes a further dampening for each period into the future. Hence, the forecast function is derived as:

$\hat{X}_{t}(m)=S_{t}\left(R_{t}^{\phi}, R_{t}^{\phi^{2}}, \ldots \ldots, R_{t}^{\phi^{m}}\right)=S_{t} R_{t}^{\sum_{i=1}^{m} \phi^{i}} 23$

If $0<\phi<1$, the multiplicative trend is damped and the forecasts approach an asymptote given by the horizontal straight line $S_{t} R_{t}^{\phi /(1-\phi)}$. The error correction form of the smoothing equation is

$$
\begin{aligned}
& S_{t}=S_{t-1} R_{t}^{\phi}+\alpha e_{t} \\
& R_{t}=R_{t-1}^{\phi}+\alpha \gamma \frac{e_{t}}{S_{t-1}}
\end{aligned}
$$

\section{II.6 Forecasting results}

Due to the fundamental importance of time forecasting in many practical situation, proper care should be taken while selecting a particular model, to estimate forecast accuracy and to compare different models. The following indicators measure the forecasting results:

Root Mean Squared Error (RMSE):

$$
R M S E=\sqrt{\frac{1}{n}} \sum e_{t}^{2}
$$

Mean Absolute Error (MAE):

$$
M A E=\frac{1}{n} \sum_{t=1}^{n}\left|e_{t}\right|
$$

Mean Percentage Error (MPE):

$$
M P E=\frac{1}{n} \sum_{t=1}^{n} \frac{e_{i}}{X_{i}} \times 100
$$

Mean Absolute Percentage Error (MAPE):

$$
\begin{aligned}
& \text { MAPE }=\frac{1}{n} \sum_{t=1}^{n}\left|\frac{e_{t}}{X_{t}}\right| \times 100 \\
& \text { III. }
\end{aligned}
$$

\section{DATA ANALYSIS}

We employed R Studio to plot, forecast time series trend and estimate the error rate. The first step in the process was to plot the series. Figure 1 shows the time series graph of Cassava production that is non-seasonal with observable trend.

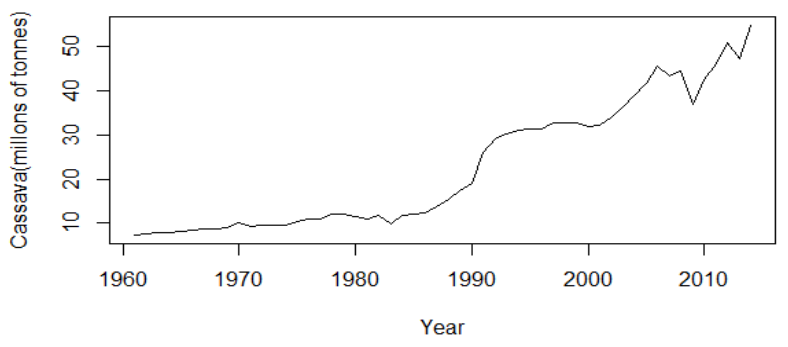

Figure 1: Time Plot of Cassava Production in Nigeria 1961-2014

The accuracy of the models: SES, HLT, ET and Holt's damped additive and multiplicative using Root Mean Square Error (RMSE), Mean Absolute Error (MAE), Mean Percentage Error (MAE), and Mean Absolute Percentage Error (MAPE); refers to as the magnitude of the error rate (errors) of an estimate, the smaller the value of these test statistics, the better the forecasts. The results of the evaluation of the forecasting models displayed in Table 1.

Table 1 Error estimation for different forecast models

\begin{tabular}{lllllc}
\hline Exponential smoothing & \multicolumn{4}{c}{ Estimated Error } & \multirow{2}{*}{ Rank } \\
\cline { 2 - 5 } & RMSE & MAE & MPE & MAPE & \\
\hline $\begin{array}{l}\text { SES } \\
\alpha=0.9999\end{array}$ & 2.4713 & 1.5525 & 3.3487 & 6.1224 & 18 \\
\hline $\begin{array}{l}\text { Holt's Linear } \\
\alpha=0.9069,\end{array}$ & 2.2928 & 1.5319 & - & 7.2117 & 13 \\
$\beta=0.0001$ & & & 2.5988 & & \\
\hline $\begin{array}{l}\text { Holt's Exponential Trend } \\
\alpha=0.8556,\end{array}$ & 2.2608 & 1.3980 & - & 5.5981 & 6 \\
$\beta=0.0001$ & & & 0.1769 & & \\
\hline $\begin{array}{l}\text { Holt's Damped Additive } \\
\alpha=0.8767, \\
\beta=0.0681, \quad \gamma=0.98\end{array}$ & 2.3353 & 1.4572 & 1.2990 & 5.6522 & 12 \\
\hline $\begin{array}{l}\text { Holt's Damped } \\
\begin{array}{l}\text { Multiplicative } \\
\alpha=0.8714,\end{array}\end{array}$ & 2.2716 & 1.4745 & - & 6.4007 & 13 \\
$\beta=0.0001, \quad \gamma=0.98$ & & & 1.4496 & & \\
\hline
\end{tabular}


The model accuracy analysis showed that Holt's with exponential trend model has the lowest ranked error rate. The trend line generated from each model in figure 2, appear to fit the data very well. For Simple Exponential Smoothing model, the estimated parameter of $\alpha$ is approximately one, as the series is clearly trending over time.

\section{Forecasts from Damped Holt's method}

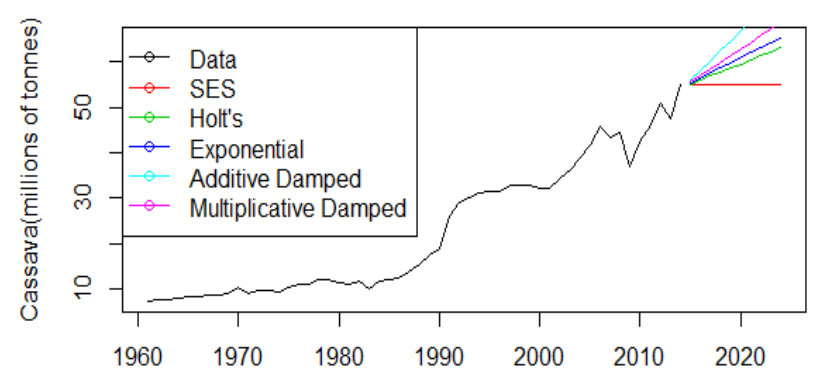

Figure 2 - 10 years forecast for all the models

\section{DISCUSSION}

From the analysis, it is evident that rising trend of Cassava production in Nigeria is not affected by seasonality. This is true as the Exponential Trend Model is the most accurate method to describe the data according to the RMSE (2.2608), MAE (1.3980), and MAPE (5.5981), while Holt's Linear is most accurate according to MPE (- 0.1769). In resolving this type of conflicting results, we selected the least ranked value of the error test statistics, which is the Exponential Trend Model.

\section{CONCLUSION}

In this study, we were able to investigate an appropriate smoothing model suitable for modeling cassava production in Nigeria. The Holt's Exponential Trend Model was found to have best described our data having the least ranked measure of error statistics of in an out of sample performance. Therefore, it is the best model in forecasting cassavas production in Nigeria.

\section{REFERENCE}

[1]. World Bank, "Agriculture in Nigeria". World Bank Published Report, 729: pp 52-68., 2010

[2]. FAO. "Cassava production" FAOSTAT statistical database retrieve from http://faostat.fao.org, 2013

[3]. Olayiwola O. O. "Short Term Market Forecast for Cassava Crops in Oyo State, Nigeria” Journal of Agriculture and Food Technology 4 (9) pp 11-18, 2014.
[4]. B Siregar, I A Butar-Butar, R.F Rahmat, U Andayani \& F Fahmi. "Comparison of Exponential Smoothing Methods in Forecasting Palm Oil Real Production", Journal of Physics: Conference Series 801012004 doi:10.1088/17426596/801/1/012004, 2017.

[5]. Fried, R.; \& George, A.C. "Exponential and Holt-Winters Smoothing", International Encyclopedia of Statistical Science, Springer Berlin Heidelberg, 2014.

[6]. Yaffe R and M. McGee. "Introduction to time series analysis and forecasting with application of SAS and SPSS" Academic Press, Inc. San Diego 2000.

[7]. Hyndman, R. J.; Anne B, Koehler J, Keith Ord and Ralph D. Snyder. "Forecasting with exponential smoothing: the state space approach", Springer-verlg, Berlin Heidelberg, 2008

[8]. Holt, C. C. "Forecasting trends and seasonal by exponentially weighted averages", O.N.R. Memorandum 52/1957, Carnegie Institute of Technology, 1957.

[9]. Gardner, Jr, E. S. and E. McKenzie. "Forecasting trends in time series", Management Science, 1985.

[10]. Taylor, J. W. "Exponential Smoothing with a Damped Multiplicative Trend", International Journal of Forecasting, 2003 